\title{
Mental health and immunity (Review)
}

\author{
CRISTIAN VASILE \\ Educational Sciences Department, Petroleum-Gas University of Ploiesti, 100680 Ploiesti, Romania
}

Received September 2, 2020; Accepted October 2, 2020

DOI: $10.3892 /$ etm.2020.9341

\begin{abstract}
The immune system consists of a complex biological and psychological network designed for fighting against infections and to protect the body from pathogen factors, including the internal ones. In the past, for a long time inflammation and infectious diseases were thought to be only the result of the genetic heritage and the biological functioning of the body, when the pathogenic factors acted within the body. Studies in recent decades stressed the importance of psychological balance and mental health on the body immunity. Psychoneuroimmunology studies indicated the thoughts and emotional patterns, and the psychological dynamics are strongly interrelated with the immune response. Moreover, the immunological mechanisms not only regulates the health of the person, but they are also an important part of the individual adaptive process in the environment. In various studies, the results of each treatment modality (drug interventions and psychosocial interventions) were observed and compared in patients with mental health problems associated with immune reactions (inflammation). Psychosocial interventions suggest increased efficiency in reducing inflammation and improving immune system function.
\end{abstract}

\section{Contents}

1. Introduction

2. Mechanisms involved in brain functioning and immune response

3. Psychosocial interventions on immune system

4. Discussion and conclusions

\section{Introduction}

In the first half of the past century, there was a popular belief, or natural knowledge, even among doctors, indicating that

Correspondence to: Professor Cristian Vasile, Educational Sciences Department, Petroleum-Gas University of Ploiesti, Bulevardul București 39, 100680 Ploiesti, Romania

E-mail: clinical_psycho@yahoo.com

Key words: mental health, psychological balance, psychoneuroimmunology, immune system, adaptation the emotion of a person influences its biological health. No scientific evidence had been analyzed at that time, but people knew that the psychological state is important in maintaining health or not.

At the beginning of the 20th century, Kraepelin and Wagner von Jauregg analyzed the role of infections and the immune system response in psychiatric disorders (1). Starting with the thirties, Hans Selye, the well-known endocrinologist, introduced the concept of stress (as general adaptation syndrome), and this way the relation between different factors influencing the body and the organic response started to be studied also from a psychological perspective. Stress is the body's reaction to a condition such as physical and psychological threats or challenges. In humans, the autonomic nervous system and the hypothalamic-pituitary-adrenal (HPA) axis are the two major systems that respond to stress (2). Psychological stress has been used in clinical trials to observe the interactions between the brain and the immune system. Stressed patients have been found to have consistent behavioral abnormalities (e.g., depressed mood and impaired sleep), along with neuroendocrine and sympathetic nervous system (SNS) dysregulations. However, the relevant mechanisms were not explored in the mentioned studies (3).

In different studies, depressed patients displayed elevated levels of central corticotropin-releasing hormone (CRH) in the central nervous system, and this neuropeptide is involved in the integration of different types of stress response: behavioral, neural, neuroendocrine and immune. High levels of $\mathrm{CRH}$ induce strong declines in innate and cellular immune responses and were related to changes in peripheral immunity (3).

Other studies have indicated that infections may be the cause of various psychiatric symptoms (e.g., mood disorders, delirium and psychotic disorders). On the other hand, cytokines (which regulate the cellular immune system and are involved in both the innate and the adaptive immune response) and an immune state which favors inflammation are involved in the pathogenesis of major depression (1). There are also studies underlying the direct relation between psychological tensions and psychosomatics (4). Even if the treatment of various mental health issues can be medication, psychotherapy, or both (5), we should consider in all these interventions the possibility that inflammation or even an infection is associated with the mental health disorder. Different problems lead to different pathological outcomes, such as dissociative identity disorders (6), specific receptor profiles for antipsychotic molecules (7), and so on. Psychosis involves the lack of 
reality testing and the appearance of strange behaviors in the patient (8), these elements being strongly related to low social and professional skills.

\section{Mechanisms involved in brain functioning and immune response}

The brain and the immune system change, in different forms, functionally relevant messages, the main function being homeostasis. These two systems communicate through complex chemical messengers that can leave their specific anatomical locations (9).

Several substances in the category of chemical messengers [small molecules, such as nitric oxide or neuroendocrine peptides, such as corticotrophin-releasing hormone (CRH); large proteins, including cytokines and growth factors with the respective receptors] also correlate these two systems (9).

Two important pathways correlate the brain and the immune system: The autonomic nervous system (ANS) through the direct neuronal circuits and the neuroendocrine pathway through the pituitary gland. ANS is mostly autonomous because its activities are not under direct conscious control. ANS works by three components: the sympathetic (noradrenergic) and parasympathetic (cholinergic) systems, which originate in the central nervous system (CNS) (cell bodies in the brainstem and spinal cord), and the enteric system, which is located in the wall of the gastrointestinal tract (9).

Studies underlined the role of inflammation in depression $(10,11)$ and have shown the existence of neural connections with lymphoid tissue (12). There are lymphocyte receptors for various neurotransmitters in addition to acetylcholine and norepinephrine. The area where, at the parasympathetic level, acetylcholine modulates several immune reactions through the vagus nerve, the sympathetic nervous system can intervene in the T helper 1 and Thelper 2 (TH1/TH2 which express different cytokine patterns) balance by stimulating the $\beta$-adrenergic receptor, for example (13).

The role of inflammation in depression has been examined in recent years in detail (14). The inflammatory immune response to stress, together with the link between stress and depression, shows that there is a correlation between inflammation and depression $(15,16)$. The risk of depression is high in different disorders with an inflammatory component: diabetes, cardiovascular disease, infections, autoimmune disorders and metabolic syndrome $(17,18)$.

DSM-III and DSM-IV discuss immune differences between subtypes of major depression. Some studies found evidence of those aspects (19). HPA dysregulation may vary in keeping with depression type $(20,21)$. Other studies evaluated the HPA axis activity (serum cortisol and ACTH) and inflammation (the proportion of the serum concentrations of interleukin-1 receptor antagonist and interleukin-1 beta) in samples of depressed patients (melancholic and non-melancholic depression). Results indicated the melancholic group had elevated measures of HPA activity relative to controls whereas the non-melancholic group had elevated measures of inflammation (22). One last study discussed here reported that interleukin-1 beta (IL-1 $\beta$ ) production in stimulated lymphocytes was inversely correlated with age-of-onset and directly correlated with duration of illness in subjects with dysthymia (23).

\section{Psychosocial interventions on immune system}

Research in the field of psychoneuroimmunology shows that the mechanisms of immunity regulation are part of a complex system of adaptive responses. This understanding of the interactions between the brain and the immune system greatly supports a deeper understanding of the mechanisms underlying health and disease, as well as the role of emotions and stress in health. The future of psychoneuroimmunological research is most likely related to a deep understanding of human immune deficiency in stress and the clinical significance of psychosocially induced changes in immune function.

Affective structures that are perceived to be stressful are accompanied by autonomic and neuroendocrine changes capable of influencing immune function and thus likely susceptibility to a variety of diseases (24). In contrast, behavioral interventions that reduce anxiety or stress decrease the intensity or duration of neuroendocrine responses and thus achieve a balance of immune function that promotes well-being and health $(25,26)$.

An analysis of mortality in 195 countries from 1980 to 2017 (27) indicated that more than 50\% of all deaths in the world today are attributed to inflammatory diseases (28). Although drug interventions are the first choice to address this serious public health problem, these interventions are often costly and can have adverse biological and clinical effects. As a result, the World Health Organization, the US National Academy of Medicine, and other large institutions have set the goal of using psychosocial interventions when possible $(29,30)$.

Studies show that there is an important ability of psychosocial interventions to enhance immunity and improve immunity-related health outcomes. These studies show that the processes of the immune system are influenced by social, neurocognitive and behavioral factors $(12,13)$.

Existing studies and meta-analyses focused mainly on one type of intervention, such as cognitive-behavioral therapy (CBT) (31), meditation $(32,33)$, mind-body interventions (34), lifestyle changes (35), body-mind therapies (36) and stress management (37).

A very recent meta-analysis (11) looked at 8 types of psychosocial intervention: behavioral therapy, cognitive therapy, CBT, CBT plus treatment (e.g., CBT plus benzodiazepines or therapeutic sessions by phone or video), supportive therapy, multiple or combined interventions, other psychotherapies and psychoeducation. At the same time, 7 results of the immune system that could be influenced by these interventions were studied: proinflammatory cytokines and markers, anti-inflammatory cytokines, antibodies, immune cell numbers, natural killer cell activity, viral load (e.g., HIV RNA) and other immune results.

The percentages resulting from the extensive processing of these data show that, compared to the control group, psychosocial interventions were associated with an $18.0 \%$ reduction in the harmful function of the immune system (11).

The above-mentioned analysis indicated that from eight interventions examined, two were significantly associated with changes in immune system outcomes: CBT (31 studies) and 
multiple or combined interventions ( 7 studies). Psychological interventions having a group component were associated with enhanced immune function, even if the statistical significance was not reached (11).

\section{Discussion and conclusions}

The present review analyzes different studies, in which the results of each treatment modality (drug interventions and psychosocial interventions) were observed and compared in patients with both immune problems (inflammation) and mental health problems. Studies in recent years and especially meta-analyses have led to the development and testing of therapeutic interventions that work effectively in treating mental health issues associated with immune problems. Psychosocial interventions suggest an increased efficiency in decreasing inflammation and improving the function of the immune system (11).

The direct link between certain mental health problems (e.g., depression) and inflammation may not have been identified so far, but the correlation between psychological stress (found in depression) and inflammation has already been demonstrated. However, in the present review we need to consider some important limitations and observations: i) Mental health problems were assessed with different instruments in different patients, and the measurement results were different depending on the specifics of these scales. ii) Patients personalities and histories are not analyzed in most studies, but they are important in the individual assessment of mental health and immunity. iii) Cultural habits (diet and substance use) must be taken into account when assessing health. The mentioned studies and especially the exhaustive meta-analysis from 2020 (11) have significant implications in the research on the importance of psychosocial interventions in the efficient functioning of the immune system while maintaining the psychological balance.

\section{Acknowledgements}

Not applicable.

\section{Funding}

No funding was received.

\section{Availability of data and materials}

All data generated or analyzed during this study are included in this published article.

\section{Authors' contributions}

CV contributed in the design of the study, data gathering, drafting the manuscript and was involved in critical revision of the manuscript for important intellectual content. CV read and approved the final manuscript.

\section{Ethics approval and consent to participate}

Not applicable.

\section{Patient consent for publication}

Not applicable.

\section{Competing interests}

The authors declare that they have no competing interests.

\section{References}

1. Müller N: Infectious diseases and mental health. In: Comorbidity of Mental and Physical Disorders. Key Issues Ment Health. Vol. 179. Sartorius N, Holt RIG and Maj M (eds). Karger, Basel, pp99-113, 2015.

2. Ulrich-Lai YM and Herman JP: Neural regulation of endocrine and autonomic stress responses. Nat Rev Neurosci 10: 397-409, 2009.

3. Irwin MR: Human psychoneuroimmunology: 20 years of discovery. Brain Behav Immun 22: 129-139, 2008

4. Trifu S, Delcuescu C and Boer CM: Psychosomatics and psychical tension (clinical research). Procedia Soc Behav Sci 33: 128-132, 2012.

5. Vasile C: CBT and medication in depression (Review). Exp Ther Med 20: 3513-3516, 2020.

6. Trifu S: Dissociative identity disorder. Psychotic functioning and impairment of growing-up processes. J Educ Sci Psychol 9: 102-108, 2019.

7. Trifu S and Trifu AD: Receptor profiles of atypical antipsychotic molecules. University Politehnica of Bucharest Scientific Bulletin series B-Chemistry and Materials Science 82: 113-128, 2020 .

8. Trifu S, Marica S, Braileanu D, Carp EG and Gutt AM: Teaching psychiatric concepts of neurosis, psychosis and borderline pathology. Conceptual boundaries. Procedia Soc Behav Sci 203: 125-129, 2015.

9. Ziemssen T and Kern S: Psychoneuroimmunology-cross-talk between the immune and nervous systems. J Neurol 254 (Suppl 2): II8-II11, 2007.

10. Ellul P, Mariotti-Ferrandiz E, Leboyer M and Klatzmann D: Regulatory $\mathrm{T}$ cells as supporters of psychoimmune resilience: Toward immunotherapy of major depressive disorder. Front Neurol 9: 167, 2018.

11. Shields GS, Spahr CM and Slavich GM: Psychosocial interventions and immune system function: A systematic review and meta-analysis of randomized clinical trials. JAMA Psychiatry e200431: Jun 3, 2020 (Online ahead of print).

12. Steinman L: Elaborate interactions between the immune and nervous systems. Nat Immunol 5: 575-581, 2004.

13. Elenkov IJ, Wilder RL, Chrousos GP and Vizi ES: The sympathetic nerve - an integrative interface between two supersystems: The brain and the immune system. Pharmacol Rev 52: 595-638, 2000.

14. Miller AH and Raison CL: The role of inflammation in depression: From evolutionary imperative to modern treatment target. Nat Rev Immunol 16: 22-34, 2016.

15. Pariante CM: Why are depressed patients inflamed? A reflection on 20 years of research on depression, glucocorticoid resistance and inflammation. Eur Neuropsychopharmacol 27: 554-559, 2017.

16. Calcia MA, Bonsall DR, Bloomfield PS, Selvaraj S, Barichello T and Howes OD: Stress and neuroinflammation: A systematic review of the effects of stress on microglia and the implications for mental illness. Psychopharmacology (Berl) 233: 1637-1650, 2016.

17. Shelton RC and Miller AH: Eating ourselves to death (and despair): The contribution of adiposity and inflammation to depression. Prog Neurobiol 91: 275-299, 2010.

18. Slavich GM and Irwin MR: From stress to inflammation and major depressive disorder: A social signal transduction theory of depression. Psychol Bull 140: 774-815, 2014.

19. Blume J, Douglas SD and Evans DL: Immune suppression and immune activation in depression. Brain Behav Immun 25: 221-229, 2011.

20. Evans DL and Nemeroff CB: The clinical use of the dexamethasone suppression test in DSM-III affective disorders: Correlation with the severe depressive subtypes of melancholia and psychosis. J Psychiatr Res 21: 185-194, 1987. 
21. Nelson JC and Davis JM: DST studies in psychotic depression: A meta-analysis. Am J Psychiatry 154: 1497-1503, 1997.

22. Kaestner F, Hettich M, Peters M, Sibrowski W, Hetzel G, Ponath G, Arolt V, Cassens U and Rothermundt M: Different activation patterns of proinflammatory cytokines in melancholic and non-melancholic major depression are associated with HPA axis activity. J Affect Disord 87: 305-311, 2005.

23. Anisman H, Ravindran AV, Griffiths J and Merali Z: Endocrine and cytokine correlates of major depression and dysthymia with typical or atypical features. Mol Psychiatry 4: 182-188, 1999.

24. Buske-Kirschbaum A, Kern S, Ebrecht M and Hellhammer DH: Altered distribution of leukocyte subsets and cytokine production in response to acute psychosocial stress in patients with psoriasis vulgaris. Brain Behav Immun 21: 92-99, 2007.

25. Davidson RJ, Kabat-Zinn J, Schumacher J, Rosenkranz M, Muller D, Santorelli SF, Urbanowski F, Harrington A, Bonus K and Sheridan JF: Alterations in brain and immune function produced by mindfulness meditation. Psychosom Med 65 564-570, 2003

26. Gaab J, Blättler N, Menzi T, Pabst B, Stoyer S and Ehlert U: Randomized controlled evaluation of the effects of cognitivebehavioral stress management on cortisol responses to acute stress in healthy subjects. Psychoneuroendocrinology 28 : 767-779, 2003

27. Roth GA, Abate D, Abate KH, Abay SM, Abbafati C, Abbasi N Abbastabar H, Abd-Allah F, Abdela J, Abdelalim A, et al: GBD 2017 Causes of Death Collaborators: Global, regional, and national age-sex-specific mortality for 282 causes of death in 195 countries and territories, 1980-2017: A systematic analysis for the global burden of disease study 2017. Lancet 392 . 1736-1788, 2018.

28. Furman D, Campisi J, Verdin E, Carrera-Bastos P, Targ S, Franceschi C, Ferrucci L, Gilroy DW, Fasano A, Miller GW, et al: Chronic inflammation in the etiology of disease across the life span. Nat Med 25: 1822-1832, 2019.

29. World Health Organization and International Initiative for Impact Evaluation: An evidence map of social, behavioural and community engagement interventions for reproductive, maternal, newborn and child health. World Health Organization, 2017.
30. National Institute of Mental Health: National Institute of Mental Health Strategic Plan for Research. National Institute of Mental Health, 2015.

31. Crepaz N, Passin WF, Herbst JH, Rama SM, Malow RM, Purcell DW, Wolitski RJ; HIV/AIDS Prevention Research Synthesis Team: Meta-analysis of cognitive-behavioral interventions on HIV-positive persons' mental health and immune functioning. Health Psychol 27: 4-14, 2008.

32. Black DS and Slavich GM: Mindfulness meditation and the immune system: A systematic review of randomized controlled trials. Ann N Y Acad Sci 1373: 13-24, 2016.

33. Pascoe MC, Thompson DR, Jenkins ZM and Ski CF: Mindfulness mediates the physiological markers of stress: Systematic review and meta-analysis. J Psychiatr Res 95: 156-178, 2017.

34. Bower JE and Irwin MR: Mind-body therapies and control of inflammatory biology: A descriptive review. Brain Behav Immun 51: 1-11, 2016.

35. Deng W, Cheung ST, Tsao SW, Wang XM and Tiwari AF: Telomerase activity and its association with psychological stress, mental disorders, lifestyle factors and interventions: A systematic review. Psychoneuroendocrinology 64: 150-163, 2016.

36. Morgan N, Irwin MR, Chung M and Wang C: The effects of mind-body therapies on the immune system: Meta-analysis. PLoS One 9: e100903, 2014.

37. Scott-Sheldon LA, Kalichman SC, Carey MP and Fielder RL: Stress management interventions for HIV+adults: A metaanalysis of randomized controlled trials, 1989 to 2006. Health Psychol 27: 129-139, 2008.

This work is licensed under a Creative Commons Attribution-NonCommercial-NoDerivatives 4.0 International (CC BY-NC-ND 4.0) License. 